Educ, 67(4), 448-458

7. Trân Văn Trường, Lâm Ngọc Ấn, Trịnh Đình Hải và công sứ (2001). Điều tra sức khỏe răng miênng toàn quốc, Nhà xuất bản Y học, Hà Nội, 67-75.

8. Vữ Manh Tuấn (2013). Nghiên cứu dự phòng sâu răng bằng Gel fluor, Luận án Tiến sĩ y học,
Trường Đai hoc $Y$ Hà Nôii.

9. Trân Thị Kim Thúy (2019). Nghiên cứu dự phòng sâu răng vĩnh viến giai đoan sớm bằng nước sức miệng flour cho học sinh 7-8 tuổi ở tỉnh Phú Thọ, Luận án tiến sỹ y học, Viện nghiên cứu khoa học y dược lâm sàng 108.

\title{
ĐÁNH GIÁ KẾT QUẢ THỤ TINH TRONG ỐNG NGHIÊMM TRÊN BÊNNH NHÂN ĐƯỢC TRƯởNG THÀNH NANG NOÃN BẰNG GnRH AGONIST TẠI BỆNH VIỆN PHỤ SẢN TRUNG ƯO'NG NĂM 2019
}

\section{TÓM TẮT}

Trong những năm gân đây GnRH agonist là thuốc được sử dụng để gây trưởng thành nang noãn thay thể hCG trong phác đồ Antagonist, kết hợp với đông phôi toàn bô và chuyển phôi đông lannh là môt giải pháp toàn diện giảm thiểu hội chứng quá kích buồng trứng trong thu tinh trong ống nghiếm. Mục tiêu: 1) Mô tả một số đặc điểm của bệnh nhân kích thích buồng trứng bằng phác đồ GnRH antagonist được gây trưởng thành nang noãn bằng $\mathrm{GnRH}$ agonist. 2) Đánh giá kết quả thụ tinh trong ống nghiệm của những bệnh nhân trên. Đối tượng và phương pháp: Nghiên cứu thực hiện trên 90 bệnh nhân thông qua hồ sơ bệnh án kết hợp phỏng vấn qua điện thoại. Kết quả: Tất cả bệnh nhân không phải nhập viện điều trị hội chứng quá kích buồng trứng (HCQKBT), chỉ ghi nhận bị quá kích buồng trứng $(13,33 \%)$ ở mức độ nhẹ. Số lượng noãn trung bình thu được là 20,91 \pm 7,13 noãn, số noãn MII chiếm tỷ lệ 77,79\%. Tỷ lệ thụ tinh trung bình đat $79,95 \pm 20,01 \%$, số phôi trung bình thu được $13,27 \pm 6,21$ phôi. Kết quả thụ tinh trong ống nghiệm được ghi nhân tốt, với tỷ thai sinh hóa đạt $55,20 \%$, tỷ lệ thai lâm sàng đạt $44,03 \%$ và tỷ lệ thai sinh sống đạt 34,33\%. Kết luận: Thụ tinh trong ống nghiệm bằng phác đồ Antagonist và trưởng thành noãn bằng GnRha cho kết quả tốt về tỷ lệ noãn trưởng thành (MII) và tỷ lệ thụ tinh tỷ lệ thai lâm sàng và tỷ lệ thai sinh sống cao. Tỷ lệ bệnh nhân quá kích buồng trứng giảm nhiều, đặc biệt không xảy ra tình trạng quá kích buồng trứng nặng.

Tư khóa: GnRH agonist, quá kích buông trứng, thụ tinh trong ống nghiệm.

\section{SUMMARY \\ EVALUATION OF THE RESULTS OF IN VITRO FERTILIZATION IN PATIENTS MATURED FOLLICLE BY USING GNRH AGONIST AT THE NATIONAL HOSPITAL OF OBSTRETICS AND GYNECOLOGY IN 2019}

${ }^{1}$ Bệnh viện đa khoa Hà Nội

${ }^{2}$ Bệnh viện phụ sản Trung Uơng

Chịu trách nhiệm chính: Nguyễn Duy Phương

Email: drnguyenduyphuong@gmail.com

Ngày nhận bài: 17.5.2021

Ngày phản biên khoa hoc: 5.7.2021

Ngày duyệt bài: 19.7.2021

\section{Nguyễn Duy Phương ${ }^{1}$, Nguyễn Xuân Họoi ${ }^{2}$}

In recent years, GnRH agonist is know as the drug used to trigger follicular maturation instead of hCG in Antagonist regimen, combined with whole embryo freezing and frozen embryo transfer (FET) is a comprehensive solution to minimize ovarian hyperstimulation syndrome (OHSS) in in vitro fertilization. Objectives: 1) Describe some features of patients stimulate ovarian with $\mathrm{GnRH}$ antagonist regimen who were triggered for follicular maturation by $\mathrm{GnRH}$ agonist. 2) Evaluate the results of in vitro fertilization of these patients. Subjects and methods: The study was carried out by descriptive retrospective method, on 90 patients based on the combination of medical records and phone interviews. Results: For all patients did not need to hospitalize for ovarian hyperstimulation syndrome (OHSS), only ovarian hyperstimulation at mild level $(13.33 \%)$ was recorded. The average number of oocytes obtained was $20.91 \pm 7.13$ oocytes, the number of matured oocytes accounted for $77.79 \%$. Additionally, the average fertilization rate was $79.95 \pm 20.01 \%$ and the average number of embryos was $13.27 \pm 6.21$ embryos. The results of in vitro fertilization were well recorded, with the biochemical pregnancy rate reaching $55.20 \%$, the clinical pregnancy rate reaching $44.03 \%$, and the live birth rate reaching $34.33 \%$. Conclusion: In vitro fertilization with an Antagonist regimen and oocyte maturation by GnRha gave good results in oocyte maturation rate, fertilization rate, clinical pregnancy rate, and live birth rate. The proportion of patients with ovarian hyperstimulation decreased significantly, especially there was no severe ovarian hyperstimulation. Keywords: GnRH agonist, ovarian hyperstimulation, in vitro fertilization.

\section{I. ĐẶT VẤN ĐỀ}

Kích thích buồng trứng trong thụ tinh ống nghiệm (TTTON) bằng phác đồ antagonist đang là lựa chọn ưu tiên số một hiện nay bởi những ưu việt vượt trội so với các phác đồ khác như thời gian kích thích buồng trứng ngắn, lượng thuốc sửa dụng ít hơn so với các phác đồ khác giúp tiết kiệm chi phí, không hình thành nang chức năng, giống với sinh lý hơn,... Trước đây hCG là thuốc mặc định dùng để gây trưởng thành nang noãn trong phác đồ trên, tuy nhiên 
đây lại được coi là tác nhân gây ra hội chứng quá kích buồng trứng (QKBT).

QKBT là biến chứng nghiêm trọng nhất của gây trường thành nang noãn trong điều trị vô sinh, hiếm muộn. QKBT sớm hoặc muộn đều gây nên những tác động không tốt đối với tình trạng sức khỏe của bệnh nhân, làm tăng thời gian và tốn kém chi phí điều trị.

Những năm gần đây, GnRH agonist được xem là thuốc đầu tay được sử dụng nhằm gây trưởng thành noãn, thay thế hCG trong phác đồ kích thích buồng trứng bằng $\mathrm{GnRH}$ antagonist đã phát triển manh trên thế giới. Phương pháp này giúp hạn chể tối đa hội chứng quá kích buồng trứng (HCQKBT) tuy nhiên có thể làm giảm tỷ lệ có thai và tăng tỷ lệ sảy thai khi chuyển phôi tươi. Việc kết hợp gầy trưởng thành nang noãn bằng GnRHa kết hợp với đông phôi toàn bộ được cho là một giải pháp toàn diện giảm thiểu HCQKBT sớm và muộn trong TTTON.

Tại Việt Nam đã có nhiều nghiên cứu đánh giá tỳ lệ QKBT cũng như chất lượng phôi, noãn ở các bệnh nhân sử dụng GnRHa trong kích thích trưởng thành nag noãn, tuy nhiên đánh giá kết quả mang thai còn rất hạn chế. Do đó, chúng tôi thực hiện nghiên cứu với mục tiêu đánh giá kết quả thụ tinh trong ống nghiệm trên bệnh nhân được gây trưởng thành nang noãn bằng $\mathrm{GnRH}$ agonist, đông phôi toàn bộ và chuyển phôi đông lạnh.

\section{II. ĐỐI TƯỢNG VÀ PHƯƠNG PHÁP NGHIÊN CỨU}

2.1 Đối tượng: 90 bênh nhân điều trị vô sinh sinh bằng phương pháp thụ tinh trong ống nghiệm được kích thích buồng trứng bằng phác đồ GnRH antagonist, gây trưởng thành nang noãn bằng GnRH agonist, đông phôi toàn bộ và chuyển phôi đông lạnh tại Trung tâm Hỗ trợ sinh sản quốc gia - Bệnh viện Phụ sản Trung ương năm 2019.

2.2 Phương pháp: Phương pháp hồi cứu mô tả. Thu thập các hồ sơ bệnh án chứa thông tin bệnh nhân có nguy cơ cao với hội chứng quá kích buồng trứng. Liên hệ với bệnh nhân thông qua số điện thoại liên hệ, thu thập thêm các thông tin về kết quả thai sau chuyển phôi và các triệu chứng lâm sàng của HCQKBT. Số liệu thu thập được thống kê bằng phần mềm Epidata 3.1 và phân tích bằng phần mềm SPSS 20.0.

\section{KẾT QUẢ NGHIÊN CỨU}

Chúng tôi nghiên cứu trên 90 hồ sơ bênh nhân làm thụ tinh trong ống nghiệm, gây trưởng thành nang noãn bằng GnRH agonist và đông phôi toàn bộ, chuyển phôi đông lạnh. Trong đó có 90 chu kỳ kích thích buồng trứng, 134 chu kỳ chuyển phôi đông lạnh, mang lại một số kết quả đáng chú ý về kết quả chuyển phôi

\subsection{Một số đặc điểm chung}

Bảng 3.1. Đặc điểm về nhóm tuối, phân loại vô sinh

\begin{tabular}{|c|c|c|c|}
\hline \multirow{2}{*}{ Nhóm tuoổi } & \multicolumn{2}{|c|}{ Phân loại vô sinh } & \multirow{2}{*}{ Tổng } \\
\cline { 2 - 4 } & Nguyên phát & Thứ phát & \\
\hline$\leq 35$ tuối & 65 & 17 & 82 \\
\hline$>35$ tuối & 5 & 3 & 8 \\
\hline Tống & $\mathbf{7 0}$ & $\mathbf{2 0}$ & $\mathbf{9 0}$ \\
\hline
\end{tabular}

Nhận xét: Phần lớn bệnh nhân nằm trong độ tuổi sinh sản, có 82 bệnh nhân (chiếm 91,1\%) từ 35 tuổi trở xuống. Nguyên nhân vô sinh nguyên phát chiếm tỷ lệ cao $(77,78 \%)$, gấp khoảng 3,5 lần tỷ lệ vô sinh thứ phát. Nhóm phụ nữ dưới 35 tuổi vô sinh nguyên phát chiếm tỷ lệ $72,22 \%$.

Bảng 3.2. Đặc điểm mức độ quá kích buồng trứng

\begin{tabular}{|c|c|c|}
\hline $\begin{array}{c}\text { Quá kích buồng } \\
\text { trứng }\end{array}$ & $\begin{array}{c}\text { Số lượng } \\
\text { (n) }\end{array}$ & $\begin{array}{c}\text { Tỷ lệ } \\
\text { (\%) }\end{array}$ \\
\hline Không quá kích & 78 & 86,67 \\
\hline Quá kích nhẹ & 12 & 13,33 \\
\hline Tống & $\mathbf{9 0}$ & $\mathbf{1 0 0}$ \\
\hline
\end{tabular}

Nhận xét: Đa số bệnh nhân không gặp tình trạng quá kích buồng trứng $(86,67 \%) .12$ trường hợp xuất hiện quá kích buồng trứng đều ở mức độ nhẹ, không có ca nào ở mức trung bình hoặc nặng. không có ca nào phải nhập viện điêuu trị.

Kết quả thụ tinh trong ống nghiệm của đối tượng nghiên cứu

Bảng 3.3. Số lượng, chât lượng noãn thu dược

\begin{tabular}{|c|c|c|c|}
\hline $\begin{array}{l}\text { Phân loại } \\
\text { noãn }\end{array}$ & $\begin{array}{c}\text { Số } \\
\text { Iượng }\end{array}$ & Tỷ lệ & $\bar{X} \pm$ SD \\
\hline Số noãn MII & 1464 & 77,79 & $16,27 \pm 7,17$ \\
\hline Số noãn MI & 162 & 8,61 & $1,8 \pm 2,12$ \\
\hline $\begin{array}{l}\text { Số noãn } \\
\text { thoái hóa }\end{array}$ & 193 & 10,26 & $2,14 \pm 3,57$ \\
\hline Số noãn GV & 25 & 1,33 & $0,28 \pm 0,70$ \\
\hline Tống & 1882 & 100 & $20,91 \pm 7,13$ \\
\hline
\end{tabular}

Nhận xét: Số lượng noãn trung bình ở bệnh nhân khá tối ưu, trung bình thu được 20,91 \pm 7,13 noãn. Trong đó, số noãn MII chiếm tỷ lệ cao, đạt tỷ lệ $77,79 \%$ với số lượng trung bình là̀ $16,27 \pm 7,17$ noãn.

\section{Bảng 3.4. Sốlướng và tỷ lê noãn thu tinh}

\begin{tabular}{|c|c|}
\hline Kết quả thụ tinh & $\mathbf{X} \pm$ SD \\
\hline Số noãn thụ tinh trung bình & $16,63 \pm 7,10$ \\
\hline Tỷ lệ thụ tinh trung bình (\%) & $79,95 \pm 20,01$ \\
\hline
\end{tabular}

Nhận xét: Số noãn được thụ tinh trung bình khá cao, đạt $16,63 \pm 7,10$ noãn. Tỷ lệ thụ tinh trung bình đạt xấp xỉ $80 \%$ số noãn thu được.

Bảng 3.5. Số lượng, chất lượng phôi thu 


được
\begin{tabular}{|c|c|c|c|}
\hline Kê̂t quả & Số lượng & Tỷ lệ & $\bar{X} \mathbf{~}$ SD \\
\hline Số phôi độ 1 & 112 & 9,38 & $1,22 \pm 2,97$ \\
\hline Số phôi độ̉ 2 & 231 & 19,35 & $2,57 \pm 2,24$ \\
\hline Số phôi độ 3 & 440 & 36,85 & $4,89 \pm 3,68$ \\
\hline Số phôi độ̂ 4 & 411 & 34,42 & $4,57 \pm 5,27$ \\
\hline $\begin{array}{c}\text { Tống sốn } \\
\text { phôi }\end{array}$ & $\mathbf{1 1 9 4}$ & $\mathbf{1 0 0}$ & $\begin{array}{c}\mathbf{1 3 , 2 7} \\
\mathbf{6 , 2 1}\end{array}$ \\
\hline
\end{tabular}

Nhận xét: Số phôi trung bình của nhóm đối tượng nghiên cứu đạt 13,27 $\pm 6,21$ phôi. Trong đó phôi độ 3 thu được chiếm tỷ lệ cao nhất (36,85\%), tiếp đó là phôi độ 4 (34,42\%). Phôi độ 1 chiếm tỷ lệ thấp nhất, chỉ có $9,38 \%$ số phôi thuộc phân loại này.

Bảng 3.6. Đánh giá kêt quả có thai

\begin{tabular}{|c|c|c|}
\hline Kết quả & $\begin{array}{c}\text { Số lượng } \\
\text { (n) }\end{array}$ & $\begin{array}{c}\text { Tỷ lệ } \\
\text { (\%) }\end{array}$ \\
\hline Thai sinh hóa & 74 & 55,2 \\
\hline Thai lâm sang & 59 & 44,03 \\
\hline Thai diền tiến & 50 & 37,31 \\
\hline Sinh sống & 46 & 34,33 \\
\hline Tống số lần chuyến phôi & 134 & \\
\hline
\end{tabular}

Nhận xét: Sau tổng số 134 lần chuyển phôi của 90 bệnh nhân, tỷ lệ thai sinh hóa đạt $55,20 \%$, tỳ lệ thai lâm sàng đạt $44,03 \%$, tỷ lệ thai diễn tiến đạt $37,31 \%$. Trung bình 3 lần chuyển phôi sẽ có 1 trẻ được sinh ra, tỷ lệ sinh sống đạt 34,33\%.

\section{BÀN LUẬN}

\subsection{Một số đặc điểm của đối tượng nghiên cứu}

Đặc điểm về tuổi. Nghiên cứu của chúng tôi cho thấy đa số bệnh nhân ở độ tuổi dưới 35 tuổi, độ tuổi trung bình được ghi nhận là 28,91 $\pm 4,52$ tuổi. Kết quả này tương đương với kết quả nghiên cứu của La Thị Phương Thảo (2016), với độ tuổi trung bình ở nhóm bệnh nhân trưởng thành noãn bằng GnRHa là $29,34 \pm 3,8$.[2] Ớ những phụ nữ trẻ tuổi thì chất lượng noãn và phôi sẽ tốt hơn, dự trữ buồng trứng tốt hơn so với phư nữ trên 35 tuổi.

Phẩn loại vô sinh. Chúng tôi thấy rằng tỷ lệ vô sinh bnguyên phát khá cao, chiếm tỷ lẹ̉ $77,78 \%$. Kết quả này cao hơn nghiên cứu của tác giả La Thị Phương Thảo tỷ lệ vô sinh nguyên phát ở nhóm gây trưởng thành nang noãn bằng GnRHa là 66\%.[2] Nhóm phụ nữ dưới 35 tuổi vô sinh nguyên phát chiếm tỷ lệ $72,22 \%$.

Tỷ lệ quá kích buồng trứng. Với mục đích tăng cơ hội thành công của một chu kỳ điểu trị vô sinh bằng thụ tinh trong ống nghiệm thì kích thích buồng trứng là một khâu rất quan trọng. Tuy nhiên, kích thích buồng trứng thường đi kèm với quá kích buồng trứng. Trong nghiên cứu của chúng tôi, tỷ lệ bệnh nhân bị quá kích buồng trứng chiếm tỷ lệ 13,33\%. Toàn bộ các trường hợp bị quá kích buồng trứng với mức độ quá kích nhẹ, không ghi nhận ca bệnh nào ở mức độ trung bình hoặc nặng tức là $100 \%$ không phải nhập viện điều trị. Một nghiên cứu của Mathilde Bourdon và cộng sự thực hiện năm 2020 cũng cho thấy sử dụng GnRha gây trưởng thành nang noãn giảm thiểu đáng kể nguy cơ quá kích buồng trứng cho bệnh nhân.[3]

Năm 2017, tác giả Thor Haahr và cộng sự thực hiện nghiên cứu phân tích trên tổng số 859 bệnh nhân, được chia thành hai nhóm: một nhóm được kích hoạt trưởng thành noãn bằng GnRHa, nhóm còn lại được can thiệp bằng hCG. Kết quả nghiên cứu chỉ ra rằng tỳ lệ quá kích buồng trứng ở nhóm $\mathrm{GnRHa}$ và hCG lần lượt là $0,9 \%$ và $1,7 \%$.[6] Có thể thây phần nào vai trò của GnRHa được sử dụng để gây trưởng thành noãn có ý nghĩa trong việc hạn chế quá kích buồng trứng của bệnh nhân.

4.2 Kết quả thụ tinh trong ống nghiệm của đối tượng nghiên cứu

Số lượng, chất lượng noãn. Trong nghiên cứu của chúng tôi, số noãn trung bình thu được là $20,91 \pm 7,13$ noãn. Số noãn thu được của bệnh nhân khá tối ưu, tương đương kết quả nghiên cứu của Giang Huỳnh Như (2012) với số noãn trung bình chọc hút được là $20,27 \pm 6,56$.[1]

Tổng số noãn thu được của các bệnh nhân là 1882 noãn, trong đó số noãn MII đạt 1464 noãn, chiếm tỷ lệ 77,79\%. Trung bình thu được 16,27 $\pm 7,17$ noã̃n MII. Kết quả này tương đương với nghiên cứu của Vương Thị Ngọc Lan và cộng sự, ghi nhận số noãn MII thu được ở ba nhóm sư dụng 3 liều triptorelin với nồng độ lần lượt là 0,2 $\mathrm{mg} ; 0,3 \mathrm{mg} ; 0,4 \mathrm{mg}$ lần lượt là $16,0 \pm 8,5 ; 15,9$ $\pm 7,8$ và $14,7 \pm 8,4$ nang.[7]

Kêtt quả thụ tinh noãn. Số noãn thụ tinh trung bình nhóm bệnh nhân nghiên cứu đạt $16,63 \pm 7,10$ noãn, tỷ lệ thụ tinh trung bình đạt $79,95 \pm 20,01 \%$ số noãn thu được. Kết quả này tương đương với kết quả của tác giả La Thị Phương Thảo, với số noãn thụ tinh trung bình đạt $16,04 \pm 6,25$ noãn ở nhóm bệnh nhân gây trưởng thành noãn bằng $\mathrm{GnRHa}$, tỷ lệ thụ tinh trung bình đạt $71,24 \%$.[2]

Số phôi trung bình thu được là $13,27 \pm 6,21$ phôi, trong đó phần lớn là phồi độ 3 và phôi độ 4. Tỷ lệ phôi độ 3 thu được là $36,85 \%$, trung bình thu được 4,89 $\pm 3,68$; trung bình thu được $4,57 \pm 5,27$ phôi độ 4 , chiếm tỷ lệ 34,42\% tổng số phôi thu được. 
Kết quả có thai. Với 134 chu kỳ chuyển phôi trên 90 bệnh nhân, số trường hợp mang thai sinh hóa được ghi nhận là $55,2 \%$. Đây là các trường hợp được xét nghiệm $\beta$ hCG 14 ngày sau chuyển phôi, được ghi nhân khi $\beta$ hCG $\geq 25$ IU/L. Tỷ lệ mang thai sinh sinh hóa trong nghiên cứu của chúng tôi cao hơn so với nghiên cứu của tác giả Iliodromiti Stamatina thực hiện năm 2013, với tỷ lệ màng thai sinh hóa được ghi nhận ở nhóm sử dụng GnRHa kích hoạt trưởng thành noãn là 36,9\% [4]. Tác giả đã thực hiện một nghiên cứu đoàn hệ hồi cứu trên 363 phụ nữ sử dunng GnRHa kích hoạt trưởng thành noãn với sự hỗ trợ steroid hoàng thể chuyên sâu và 257 phụ nữ sử dụng hCG thông thường. Phụ nữ có nguy cơ quá kích buồng trứng được xác định bằng tiêu chí số nang trứng $\geq 15$ nang có kích thước $\geq 12$ $\mathrm{mm}$ vào ngày kích hoạt.

Kết quả nghiên cứu của chúng tôi cho thây tỷ lệ thai lâm sàng là 44,03\%. Một nghiên cứu của tác giả Giang Huỳnh Như thực hiện năm 2012 cho kết quả tương đương với chúng tôi, khi tỷ lệ mang thai lâm sàng là $45,5 \%$, tuy nhiên ở nghiên cứu này, tất cả bệnh nhân được chuyển phôi tươi [1]. Một nghiên cứu của Itai Bar Hava cho thấy tỷ lệ mang thai lâm sàng tương đương, đạt 45,1\%.[5]

Kết quả có thai lâm sàng của chúng tôi lớn hơn kết quả của tác giả Iliodromiti Stamatina với tỷ lệ đạt $30 \%$ ở nhóm sử dụng GnRH kích hoạt trưởng thành noãn.[4]

Tỳ lệ thai diễn tiến được chúng tôi phân tích đạt 37,31\%, Kết quả này tương đương với nghiên cứu của tác giả Itai Bar Hava và cộng sự, tỷ lệ thai diễn tiến được ghi nhận là 39,2\%.[5]

Một nghiên cứu khác của tác giả Youssef (2010) cho thây tỷ lệ thai diễn tiến thấp hơn nghiên cứu của chúng tôi. Tác giả đã tiến hành một nghiên cứu cứu gộp 11 nghiên cứu thử nghiệm lâm sàng ngẫu nhiên có đối chứng so sánh tác dụng gây trưởng thành noãn của GnRHa với hCG trên bệnh nhân được kích thích buồng trứng bằng phác đồ antagonist, cho thấy tỷ lệ thai diễn tiến ở nhóm sử dụng GnRHa là $12 \%$ đến 22\%.[8]

Kết quả của chúng tôi cho thấy, tỷ lệ sinh sống đạt $34,33 \%$. Tỷ lệ sinh sống này cao hơn kết quả được Iliodromiti Stamatina nghiên cứu, với tỷ lệ đạt 29,8\%.[4] Nghiên cứu của tác giá Thor Haahr (2017), ghi nhận tỷ lệ sinh sống thấp hơn, đạt 26,1\% [6]. Tỷ lệ này tương đương với nghiên cứu của Itai Bar Hava (2020) với tỷ lệ sinh sống đạt 27,45\%.[5]

\section{KẾT LUẬN}

Thụ tinh trong ống nghiệm bằng phác đồ Antagonist và trưởng thành noãn bằng $G n R h a$ cho kết quả tốt về tỷ lệ noãn trưởng thành (MII) và tỷ lệ thụ tinh tỷ lệ thai lâm sàng và tỷ lệ thai sinh sống cao. Tỷ lệ bệnh nhân quá kích buồng trứng giảm nhiều, đặc biệt không xảy ra tình trạng quá kích buồng trứng nặng.

\section{KIẾN NGH!}

Nên phổ biến áp dụng dùng GnRH agonist để trưởng thành nang noãn, đông phôi toàn bộ và chuyển phôi đông lạnh cho các bệnh nhân làm IVF có nguy cơ cao với HCQKBT khi kích thích buồng trứng bằng phác đồ antagonist. việc sử dụng thuốc này giảm thiểu tối đa HCQKBT đồng thời cho kết quả noãn, phôi và có thai tương đương với các nhóm thuốc khác.

\section{TÀI LIÊU THAM KHẢO}

1. Giang Huỳnh Như, Vương Thi Ngoc Lan (2016). Sư dụng GnRH đồng vận thay thế hCG trong khởi động trưởng thành noãn ở các chu kỳ kích thích buồng trứng bằng phác đồ $\mathrm{GnRH}$ đối vân.Y Hoc TP. Hồ Chí Minh, Tập 16, Phụ bản của Số 1,175 - 179.

2. La Thị Phương Thảo (2016). So sánh hiêu quả phòng ngừa hội chứng quá kích buồng trứng và chất lượng noẩn của phác đồ gây trưởng thành noãn bằng GnRH agonistvà hCG, Luận văn Thạc sĩ y học, Đại học Y Hà Nội.

3. Mathilde Bourdon, Maeliss Peigné, Celine Solignac et al (2020). The new standard for ovulation triggering should be gnrh agonist over hcg during controlled ovarian stimulation for ivf/icsi: a systematic review and meta-analysis. Abstract Only, 114(3),163-164.

4. Iliodromiti $S$, Lan V.N, Tuong H.M et al (2013). Impact of GnRH agonist triggering and intensive luteal steroid support on live-birth rates and ovarian hyperstimulation syndrome: a retrospective cohort study. J Ovarian Res, 6, 93.

5. Itai Bar Hava, Hadar Yafee, Yeela Omer et al (2020). GnRHa for trigger and luteal phase support in natural cycle frozen embryo transfer - A proof of concept study. Reprod Bio,20(3),282-287.

6. Thor Haahr, Matheus Roque, Sandro $C$. Esteves, Peter Humaidan (2017). GnRH Agonist Trigger and LH Activity Luteal Phase Support versus hCG Trigger and Conventional Luteal Phase Support in Fresh Embryo Transfer IVF/ICSI Cycles-A Systematic PRISMA Review and Meta-analysis. Frontiers in Endocrinology.

7. Vuong T.N (2016). Gonadotropin-releasing hormone agonist trigger in oocyte donors co-treated with a gonadotropin-releasing hormone antagonist: a dose-finding study. Fertil Steril, 105(2), 356-63.

8. Youssef M.A (2010). Gonadotropin-releasing hormone agonist versus HCG for oocyte triggering in antagonist assisted reproductive technology cycles. Cochrane Database Syst Rev, 2010(11), cd008046. 